Crowding in children's visual acuity tests - effect of test design and age Running Head: Crowding and children's vision tests

Yvonne Norgett BSc(Hons) MCOptom*, John Siderov MScOptom, PhD FAAO

\author{
Anglia Vision Research, \\ Department of Vision and Hearing Sciences \\ and Vision and Eye Research Unit, \\ Postgraduate Medical Institute \\ Anglia Ruskin University
}

${ }^{*}$ Corresponding author

Email: yvonne.norgett@anglia.ac.uk

Phone +44 (0) $1223363271 \times 2671$

Fax $+44(0) 1223417712$

Anglia Ruskin University

East Rd

Cambridge

CB1 1PT

UK

Figures: 3

Tables: 2

Conflict of interest: None

Submitted: 1 December 2010 


\section{Crowding in children's visual acuity tests - effect of test design and age}

\section{Purpose}

The aim of this study was to investigate the effect of test design (crowding) and age on visual acuity in a sample of young children.

\section{Methods}

Vision was measured in 103 children aged between 4 to 9 years using 5 different visual acuity tests. The tests included 3 crowded tests: the logMAR Crowded Test, the Sonksen logMAR Test and the Crowded Kay Picture Test, and 2 single optotype tests: the single Kay Picture and Sheridan Gardiner tests. Tests were presented in a random order using standardized instructions and a defined end-point. Results were analysed in 2 age groups, younger (4-6 years) and older (7-9 years).

\section{Results}

In both groups, there was a significant main effect of test on acuity (younger: $F=63.92, d f=4, p<0.001$; older: $F=63.59, d f=4, p<0.001)$. In the younger group, an effect of crowding was seen in all three crowded tests, but in the older group, an effect of crowding was seen only in the crowded letter tests. In both groups, mean acuity was lowest with the logMAR Crowded Test, which has the closest inter-optotype spacing (0.5), slightly higher with the Sonksen Test (with inter-optotype spacing of 1.0) and highest with the single optotype tests (no crowding). More crowding was seen in the younger children. 


\section{Conclusions}

Our results show that maturation of line acuity is still taking place between the ages of 4 and 9 . Measured acuity is affected by the amount of contour interaction induced by the type of optotype (letter or picture) and by the interoptotype separation. Another factor, probably a maturation of gaze control or selective attention is responsible for the reduction in crowding with age. 
Measurement of visual acuity in children is used to detect refractive error and amblyopia and to monitor improvement of vision with treatment. Children from about 2-3 years of age are capable of performing recognition acuity tests where letters or pictures are named or matched. ${ }^{1}$ In pre-school children, visual acuity has traditionally been assessed by using isolated letter or picture optotypes; ${ }^{2,3}$ however such methods do not take account of crowding. ${ }^{4-6}$

Flom and colleagues ${ }^{7}$ described crowding as a combination of the effects of contour interaction, relative inaccuracy of gaze control and attentional factors. Contour interaction is that specific effect that occurs as a result of the close proximity of contours near an acuity target ${ }^{8}$ and is often used interchangeably (though always not correctly), with the term crowding. ${ }^{9}$ Contour interaction can be quantified in terms of its extent (the distance over which the effect is present) and its magnitude (the effect on threshold or performance). The gaze control aspect of crowding describes a relative inaccuracy of eye movements when fixating along a line of letters, possibly due to the physiological limits to motor control. Such inaccuracies may contribute to the reported decrease in visual acuity when assessing some patients using line or full letter tests. ${ }^{10}$ Lastly, the attentional effect of crowding is a cognitive feature which arises as a result of the perceptual difficulty of separating a target from surrounding features in a complex array..$^{11,12}$

In order to avoid the over-estimation of acuity which arises from single optotype tests 2, 3, 9 and to make acuity tests more sensitive to amblyopia detection, newer children's vision tests have been designed to induce crowding. A number of such tests have been produced using letter optotypes, including the logMAR Crowded Test, ${ }^{13}$ the Sonksen logMAR Test ${ }^{14}$ and the HOTV test ${ }^{15}$ as well as crowded versions of the Kay Picture Test ${ }^{16}$ and Lea Symbols ${ }^{17}$ using symbol optotypes. However, the overall level of crowding may differ between tests because of the lack of standardisation of 
the individual components of crowding. For example, surrounding a single letter with flanking bars close to the optotype induces contour interaction, thereby impairing recognition, but may not require the same level of gaze control accuracy required to read a series of letters along a line. Also the separation of optotypes from each other and from the surround bar is not standard. The ETDRS Test uses a separation of 1.0 letter width, ${ }^{18}$ which has also been used in the Sonksen Test, but the LogMAR Crowded Test uses a separation of 0.5 letter widths.

Another important but somewhat less extensively studied factor in the visual assessment of children is the effect of age on crowding. Atkinson and Braddick, used a Landolt $\mathrm{C}$ target surrounded by a circular array of Cs and Os at a fixed interoptotype spacing to compare 'crowded' with single optotype acuity. In normal 5 year olds, the resultant 'crowded' acuity, with a fixed level of contour interaction, was found to be only $58 \%$ that of adults. ${ }^{19}$ Using a target letter surrounded by four other letters, again at a fixed inter-letter spacing, Atkinson et al $^{20}$ found the ratio between the surrounded letters and the single letters in 5-7 year olds was similar to adults, but significantly greater in 3-4 year olds. Possible sources of the crowding measured in these studies are the contour interaction from the surround optotypes and the increased level of difficulty of the task in naming the surrounded optotype compared to the single optotype target. Manny, Fern and Loshin ${ }^{5}$ isolated the effect of contour interaction in 12 pre-schoolers by plotting the recognition thresholds of a $\mathrm{C}$ target with flanking bars of varying separations. Although individual variation was noted, they found that contour interaction in 3-4 year old children was quantitatively similar to adults. ${ }^{7}$ Kothe and Regan ${ }^{10}$ compared single optotype acuity with Snellen acuity and repeat letter acuity in 90 children aged 4-11. Separation of letters was one letterwidth in both charts, however, in their Snellen chart, there was a large vertical separation between lines lower down the chart and the end letters were exposed on one side, whereas in the repeat letter chart, the target letters were surrounded on all 
four sides. The line chart required accurate gaze control as well as having variable contour interaction whilst the repeat letter chart did not require accurate gaze control, but had constant contour interaction. They found that some children performed better on the repeat letter chart than the line chart, suggesting that poor control of gaze was a factor in the reduced visual acuity of these children, whilst others performed better on the line chart, suggesting that contour interaction was, for these children, the principal cause of crowding. Kothe and Regan also concluded that both uncrowded and crowded acuity improved in parallel between the ages of 4 and 11 . This finding of the continued development of uncrowded acuity beyond the age of around 6 conflicted with the conclusions of some previous authors, ${ }^{21-23}$ who reported high levels of single optotype (uncrowded) acuity at young ages with little development thereafter (see also review ${ }^{24}$ ). Semenov et $a^{25}$ found uncrowded acuity to mature at around 7 years of age. They also measured the spatial extent (zone of inhibition) of contour interaction in children aged 3-9 using a Landolt $\mathrm{C}$ target and flanking bars and found a decrease in the spatial extent of contour interaction with age, from nearly 1.7 optotype units in the younger children, reaching adult levels of 1 optotype width (or 5 times the MAR) by age 9 . In a recent study, Jeon et al ${ }^{26}$ investigated single letter and contour interaction thresholds in 5, 8 and 11 year olds with adult controls, using a Sloan E target with and without flanking bars. They found that uncrowded acuity matured to adult levels between 5 and 8 years, but that the distance over which contour interaction occurred was similar in the 3 groups of children (around 1.5 optotypes widths) but significantly greater than the distance over which an effect was found in adults (0.6 optotypes widths).

Differences in experimental methods in the above studies have led to a range of conclusions being drawn about development of crowding in children. We can however conclude that contour interaction is present in children and there is some agreement ${ }^{25,26}$ that the zone over which it occurs is greater in children than adults. 
Published normative data for the acuity with the HOTV test ${ }^{27}$ and the Sonksen LogMAR Test ${ }^{28}$ show crowded acuity continuing to improve until 6 and 8 years respectively and such data are valuable to clinicians. However, without a corresponding measure of uncrowded acuity, those studies are not able to inform the discussion about relative maturation of crowded and uncrowded acuity.

The purpose of this study was therefore to look for an age effect of crowding in line acuity tests and to investigate the effect of

(i) using different letter separations

(ii) using a picture optotype test (Kay Pictures Test) rather than letter optotypes. Visual acuity was measured in a sample of primary school children using: the logMAR Crowded Test (Keeler UK), the Sonksen logMAR Test, the Kay Picture Crowded logMAR and the Kay Picture Single logMAR Tests and the Revised Sheridan Gardiner Test. Our results showed a significant difference in visual acuity as a function of test and age, and that not all crowded tests performed the same.

\section{Methods}

\section{Participants}

School children, aged 4-9, were recruited from a primary (elementary) school in Cambridgeshire, UK. For analysis, the children were considered in two groups. Children in the younger group (39 participants) were aged between 4 years 10 months and 6 years 9 months, mean 5 years 9 months. Children in the older group (64 participants) were aged between 7 years 9 months and 9 years 8 months, mean 8 years 7 months. The number of participants in each group was sufficient to obtain a power of $80 \%$ at the $5 \%$ level (two-tailed) for an effect size of $0.1 \log$ MAR. An equal number of children in each age group were invited to participate in the study, but a greater number from the older group responded and for reasons of equity, were included. Written informed consent from the children's parents or guardians and 
verbal consent from the children was obtained before any data were collected. All children with a completed consent form who were available on the day of testing participated, the only exclusions from the results were children unable to co-operate with the testing protocol (3 did not comply). Approval of the study protocol was given by our Institutional Research Ethics Committee and the study followed the tenets of the Helsinki Declaration.

Insert Figure 1 and Table 1 about here

Visual Acuity Tests: design and scoring

The tests used in the study are depicted in Figure 1 and a summary of their main features is shown in Table 1. Each of the three crowded tests comes in booklet form with 4 optotypes in a line and a surround bar on each page. Scoring is in logMAR. The two single optotype tests are also in flip books with one optotype per page and no surround bar. To facilitate comparisons between the tests, the following modifications to the recommended testing protocol were introduced. Scores for the Sheridan Gardiner Test were converted to the nearest logMAR and letter-by-letter scoring was used. Where only 3 different optotypes of a given size were available in the single optotype tests, one of these was shown a second time. Thus, for every test, children were presented with 4 optotypes at any one size. Both Kay Picture tests are designed for use at $3 \mathrm{~m}$, but to avoid the effects of truncation, they were performed at $6 \mathrm{~m}$ and the scores modified accordingly. Thus, the LogMAR Crowded and the Sonksen Tests were viewed directly at $3 \mathrm{~m}$ and the Sheridan Gardiner and the two Kay Pictures Tests were viewed through a front-surface, optical quality mirror at $3 \mathrm{~m}$, with the examiner standing beside the child.

\section{Protocol}


Testing took place in the school hall and adequate illumination for visual acuity testing was used. Before testing, the children were familiarized with the Kay Picture optotypes. The right eye of each child was tested, using occluding glasses for the left, and spectacles were worn if they had been prescribed. The five tests were presented to each child in a random order. For each test a starting point of logMAR $0.2(6 / 9.5)$ was used and the children were asked to name the letters or pictures presented. For the line tests, children were asked to name each optotype in order from left to right. If the 0.2 line was not read correctly on initial presentation, larger lines were presented until all four optotypes were read correctly. Cards of smaller optotypes were presented and testing continued until three or more optotypes at one acuity level were named incorrectly. If a child was hesitant, they were encouraged once to guess. Visual acuity data were converted into logMAR with each correctly read optotype assigned a score of 0.025 . Each child was assessed in a single session and testing was carried out by three experienced optometrists, the two authors and a colleague from the University Eye Clinic at Anglia Ruskin University. Five children whose measured acuity was worse than logMAR $0.2(6 / 9.5)$ were referred for a full eye examination; however, data from these children were included in the study.

\section{Results}

Table 2 shows the mean logMAR and standard deviation for each test separated into the younger and older age groups. These data were then normalized to highlight the crowding effect by subtracting the unflanked single optotype logMAR results (either Sheridan Gardiner or Kay Pictures, as appropriate) from the logMAR results of the respective crowded tests. The results are plotted in Figure 2 where normalized logMAR is shown for each test and logMAR values greater than zero are indicative of crowding. The top panel shows results for the younger children and the bottom panel the older children. The data were subject to one-way repeated measures ANOVA 
and post-hoc testing was performed, where appropriate, using the Tukey test (Statistica ${ }^{\mathrm{TM}}$, Statsoft, Tulsa USA).

\section{Younger children}

There was a significant main effect of test on acuity $(F=63.92, d f=4, p<0.001)$. Mean acuity was poorest with the logMAR Crowded Test and best with the single optotype tests with the mean from the Sonksen test falling in-between. Mean acuity using the Crowded Kay Picture Test was poorer than the single optotypes tests, but better than the crowded letter tests. Post-hoc testing showed that the logMAR Crowded Test gave significantly different results to all the other tests $(p<0.001)$, as did the Sonksen Test $(p<0.05)$ and the Crowded Kay Picture test $(p<0.05)$. There was no difference between the Sheridan Gardiner and Single Kay Picture tests in this age group (Fig. 2).

Insert Figure 2 and Table 2 about here

\section{Older children}

In the older children, there was a significant main effect of test on acuity ( $F=63.59$, $d f=4, p<0.001)$. Mean acuity was poorest with the logMAR Crowded Test $(p<0.001)$. Mean acuity was best with the single optotype tests, which were not significantly different from each other. The mean acuity with the Sonksen Test fell between the logMAR Crowded Test and the single optotype tests and was significantly different to all the other tests $(p<0.001)$. Surprisingly, in the older children, mean acuity with the Crowded Kay Picture Test was no different to that from the single optotype tests (Fig. 2).

Figure 3 plots the logMAR for each of the tests as a function of age. The straight lines represent linear regression fits to each data set (acuity test). The top panel shows results of the Sheridan Gardiner (closed symbols and solid line), the Sonksen 
(open squares and dashed line) and the logMAR Crowded Tests (cross symbols and dotted line). The bottom panel shows the results of the Single Kay Pictures (closed symbols and solid line) and the Crowded Kay Pictures (open symbols and dotted line). The slopes of the regression lines for both the uncrowded, single optotype tests were not significantly different from zero $(p>0.05)$. However, the slopes were significantly different from zero for all three crowded tests (LMC $p<0.01$, Sonksen $\mathrm{p}<0.05, \mathrm{CK} p<0.01)$.

Insert Figure 3 about here

\section{Discussion}

The general level of visual acuity in our sample is consistent with published age norms ${ }^{28}$ and despite not screening for refractive error, the mean Sheridan Gardiner (i.e. single letter) acuity in both younger and older groups of children was greater than 6/5 (logMAR -0.1, Table 2). Our results are also consistent with previous studies reporting that uncrowded vision tests are easier for children to perform than crowded vision tests. $^{2,3,29,30}$ Although visual acuity was poorest with the logMAR Crowded Test in both younger and older children, younger children exhibited a greater loss in visual acuity relative to the Sheridan Gardiner results. There was also a significant difference in visual acuity between the Sonksen and Sheridan Gardiner tests for both groups (albeit not as large) and once again the younger children exhibited a greater loss with the Sonksen test relative to the Sheridan Gardiner results. These results show that while both groups of children exhibited poorer visual acuity with the crowded visual acuity tests, the two crowded letter tests used were not equally effective at inducing crowding and the crowding effect was greater in the younger group of children suggesting an age dependent effect.

Results using the Kay Picture optotypes were generally similar; the Crowded Kay Picture Test resulted in poorer visual acuity when compared to the Single Kay Pictures but only for the younger children. There was no significant difference in 
visual acuity between the Crowded Kay Pictures Test and the Single Kay Pictures in the older children, indicating that in older children the Crowded Kay Picture Test did not induce significant crowding (Fig. 2).

We used two different testing distances, $3 \mathrm{~m}$ and $6 \mathrm{~m}$, depending on the test. In testing young children, the closer $3 \mathrm{~m}$ distance enhances rapport and helps maintain attention; $;^{14,20,31}$ however, as our $6 \mathrm{~m}$ testing distance used a mirror, the examiner could stand beside the child and hence maintain the advantages of proximity to the child. There is some evidence that a near testing distance provides some advantages, ${ }^{32,33}$ although Atkinson et $a^{20}$ found no significant difference in either single or multiple letter acuity, or in the crowding effect when measured at $3 \mathrm{~m}$ and $6 \mathrm{~m}$ in 3-4 year olds. It is possible that for some of our participants, the $3 \mathrm{~m}$ testing distance conferred a small advantage for the LogMAR Crowded Test and the Sonksen Test, the effect of which would be to underestimate the crowding in these tests.

Our single optotype results, showing no effect of age in the range used (4-9 years), suggest that uncrowded acuity is mature at an earlier age than crowded acuity. This accords with the conclusions of Jeon et al ${ }^{26}$ and Semenov et al. ${ }^{25}$ However, the age at which maturity of single optotype acuity occurs differs between the studies. We found no improvement in uncrowded acuity between our younger and older children, whereas Jeon et al found a significant improvement from the ages of 5 to 8 .

Comparison of acuity results showed that our younger children had better acuity than those similarly aged children in the study by Jeon et al, where the mean logMAR of their 5 year olds was around 0 , their fig. 3 (E target), compared to our -0.18 (Sheridan Gardiner). Differences in the targets could be a possible explanation, and the fact that all the children in our study were at school and used to reading letters, whereas the orientation discrimination of the letter $E$ in the Jeon study may represent 
a more difficult task. Bondarko and Semenov ${ }^{34}$ showed the $E$ target to be generally more difficult for children compared to the Landolt C. Our results also suggest that crowded and uncrowded acuity do not develop in parallel (Fig. 3). Although this appears to conflict with the conclusions of Kothe and Regan ${ }^{10}$ (see introduction), the line acuity tests in our study have more contour interaction than the Snellen test used by Kothe and Regan, therefore making our line tests harder.

The improvement in crowded visual acuity with age (Fig. 3) is likely to reflect the development of underlying factors that contribute to the total crowding effect; the influence of contour interaction, the effect of gaze instability and attentional factors. ${ }^{9}$ On this basis, the decrease in crowding with age could result from a change in the magnitude and/or extent of contour interaction, better control of gaze, or a maturation of attention and general cognitive abilities with age or some combination of all three factors.

There is evidence that the shape of the contour interaction function in young children is similar to that of adults, with the maximum effect occurring at a similar targetflanker separation distance in children and adults. ${ }^{5}$ Studies which investigated the furthest distance of flanker from target at which an effect can be measured ${ }^{25,26}$ have shown that contour interaction occurs over larger distances in children than in adults. This finding helps to explain differences in acuity between the two crowded letter tests used in our study. The LogMAR Crowded test which resulted in the poorest acuity has the closer inter-optotype separation (0.5 letter widths) and, therefore, more contour interaction than the Sonksen Test which has an inter-optotype spacing of one letter width. The mean difference of 0.07 logMAR between these two tests (in both age groups) is greater than the mean difference in visual acuity found in adults when the inter-letter separation is changed from 1.0 to 0.5 letter- widths. ${ }^{35}$ Our finding thus supports the hypothesis that contour interaction has a greater effect in children 
than in adults. A surprising result was that the Crowded Kay Pictures Test gave significantly better mean acuity than the LogMAR Crowded Test, despite a similar inter-optotype spacing of 0.5 . It is possible that the Kay Picture optotypes do not induce as much contour interaction as letters for the same inter-optotype separation, which may also explain previous results where Crowded Kay Pictures were found to be slightly easier than letter acuity tests. ${ }^{16,36}$ One of the factors governing the extent to which optotypes induce contour interaction has been shown to be their similarity with the target optotype ${ }^{37}$ and it could be that the Kay picture optotypes are dissimilar enough from each other not to exhibit contour interaction in the same way as letter optotypes. Additionally, contour interaction is governed by the leading edge of a distracter ${ }^{9}$ and in using pictures as optotypes, there will be variation in the shape of the edge of the picture; not all will have a strong leading edge, such as a vertical line. Contour interaction in picture optotypes was successfully demonstrated by Mayer and Gross ${ }^{38}$ who modified the Allen Pictures optotypes by adding distraction bars and demonstrated crowding in isolated, surrounded pictures. However, they used a separation between optotype and distracter of between 0.1 and 0.2 times the picture size, a closer separation than used in the Crowded Kay Pictures Test.

Based on recent evidence that there is no significant change in the extent of contour interaction across an age range similar to one we used, ${ }^{26}$ the difference in mean acuity between the LogMAR Crowded Test and the Sonksen Test may be explained by contour interaction; however, an alternative explanation is needed for the agerelated improvement in crowded line acuity. In the study by Jeon et $a l,{ }^{26}$ optotype recognition did not require sequential fixation from one optotype to the next along a line (as they used single optotypes). On this basis, the decrease in crowding found with the acuity tests in our study might be explained by underlying development of more accurate gaze control in children and the development of fixational eye 
movements. There is some evidence from direct measurement of children's eye movements that fixational stability is immature in young children; ${ }^{39,40}$ however this does not constitute evidence that the retinal smear from poor fixation is the cause of reduced acuity. Kothe and Regan ${ }^{10}$ proposed that failure of some normally sighted young children to achieve adult levels of line acuity may be attributable to a delayed development of gaze selection rather than cognitive factors or contour interaction. Their evidence came from the finding that some $4-5$ year olds had better acuity measured on a repeat letter chart, which minimizes the requirement for accurate gaze control, than on a Snellen chart.

The influence of gaze control may provide another possible explanation for the relatively lower levels of crowding found using the Crowded Kay Pictures Test. The sizing of the Kay Picture optotypes was developed empirically to give an acuity equivalent to that of a Snellen chart. ${ }^{41}$ However, presumably because of their relative complexity or unfamiliarity, the Kay Picture optotypes are twice the size of the corresponding letter optotype at a given acuity level. Therefore, Kay Pictures spaced at 0.5 inter-optotype separation will have twice the angular separation as letter optotypes at the same acuity level. A young child reading a row of optotypes just above their threshold acuity and near their physiological limit of gaze control may find the picture test easier than the equivalent letter one because of the greater angular separation of the optotypes.

Despite ensuring that all of our tests extended to logMAR $-0.3(6 / 3)$, a possible truncation effect may have occurred. Where a smaller line $(-0.4)$ was available in the Crowded Kay Pictures Test, some of the children achieved one or more optotypes of this size. Therefore it is possible that truncation of acuity could have occurred for some children with exceptionally good acuity. In those cases, in all tests where there may have been a truncation effect, we re-analysed the data after assigning an 
additional two optotypes to any child whose results may have been adversely affected by a truncation effect. Our re-analysed results of the linear regression still showed that the slopes of the two single optotype tests were not significantly different from zero, as depicted in figure 3, whilst for all three crowded tests the slopes were significantly different from zero $(p<0.05)$. ANOVA and post-hoc testing gave similar results to before, the only difference with the remodelled data being that in the younger children, acuity from the Crowded Kay Pictures Test was not significantly different to the Sonksen Test $(p=0.10)$. Nevertheless, we feel that any truncation effect present was small and therefore our main findings and conclusions are not altered.

The findings of our study are consistent with the hypothesis that the reduction in crowding with age is attributable to either immature oculomotor control or to cognitive or attentional limitations. Whilst the contribution of 'attention' to crowding has been shown to be less with foveal compared to peripheral viewing, ${ }^{11}$ the mechanisms of selective attention in children are thought to be less mature in children than in adults. ${ }^{34}$ The behavioural response of children when faced with a recognition task near their threshold of acuity may also vary with age. When a test is perceived as being more difficult, a child may refuse to respond, whereas an adult may attempt the task thereby improving their score.

In conclusion, our results are consistent with the literature, showing that, in general, single optotype tests, letter or picture, overestimate visual acuity compared to crowded acuity tests. However, crowding in the Crowded Kay Pictures test is less robust than in letter tests with a similar format, which may reduce sensitivity of the Crowded Kay Pictures compared to letter tests particularly if used in older aged children. The results show poorer mean acuity using the crowded tests in the younger children and, given that no change in the single optotype acuity was shown 
across the age range, this indicates that in normally sighted children, there is still maturation of line acuity taking place between the ages of 4 and 9 . This maturation is likely to be a result of an improvement in gaze control or a maturation of selective attention or cognitive factors. As crowded tests are used to measure progress of amblyopia treatment, it is important to understand whether improvement in visual acuity over time is as a result of the treatment or merely because of an age-related reduction in crowding.

\section{Acknowledgements}

The authors thank: the pupils, parents and staff of Meridian Primary School, Comberton, UK for their enthusiastic co-operation; optometrist, Katie Miller and optometry students Emma Close, Yagmur Gullu and Faizal Malji for their help with data collection.

This work was funded in part by a grant from the College of Optometrists (UK) iPRO Small Grants Award Scheme.

This paper was presented at the American Academy of Optometry Meeting, San Francisco on $17^{\text {th }}$ November 2010. 
1. Kvarnström G, Jakobsson P. Is vision screening in 3-year-old children feasible? Comparison between the Lea Symbol chart and the HVOT (LM) chart. Acta Ophthalmologica Scandinavica 2005;83:76-80.

2. Hilton AF, Stanley JC. Pitfalls in testing children's vision by the Sheridan Gardiner single optotype method. British Medical Journal 1972;56:135.

3. Youngson RM. Anomaly in visual acuity testing in children. The British Journal of Ophthalmology 1975;59:168.

4. Levi DM, Carney, T Crowding in Peripheral Vision: Why Bigger Is Better. Current Biology 2009;19:1988-93.

5. Manny RE, Fern KD, Loshin DS. Contour interaction function in the preschool child. American Journal of Optometry and Physiological Optics 1987;64:686.

6. Stuart JA, Burian HM. A study of separation difficulty. Its relationship to visual acuity in normal and amblyopic eyes. American Journal of Ophthalmology 1962;53:471.

7. Flom MC, Weymouth FW, Kahneman D. Visual resolution and contour interaction. Journal of the Optical Society of America 1963;53:1026-32.

8. Danilova MV, Bondarko VM. Foveal contour interactions and crowding effects at the resolution limit of the visual system. Journal of Vision 2006;7:25.1.

9. Flom MC. Contour interaction and the crowding effect. Problems in Optometry 1991;3:237-57.

10. Kothe AC, Regan D. The component of gaze selection/control in the development of visual acuity in children. Optometry \& Vision Science 1990;67:770. 11. Leat SJ, Li W, Epp K. Crowding in central and eccentric vision: the effects of contour interaction and attention. Investigative Ophthalmology \& Visual Science 1999;40:504. 
12. Wolford G, Chambers L. Lateral masking as a function of spacing. Perception \& Psychophysics 1983;33:129-38.

13. McGraw PV, Winn B. Glasgow acuity cards: a new test for the measurement of letter acuity in children. Ophthalmic and Physiological Optics 1993;13:400-.

14. Salt AT, Wade AM, Proffitt R, Heavens S, Sonksen PM. The Sonksen logMAR Test of Visual Acuity: I. Testability and reliability. Journal of AAPOS 2007;11:589-96.

15. Holmes JM, Beck RW, Repka MX, Leske DA, Kraker RT, Blair RC, Moke PS, Birch EE, Saunders RA, Hertle RW. The amblyopia treatment study visual acuity testing protocol. Archives of Ophthalmology 2001;119:1345.

16. Jones D, Westall C, Averbeck K, Abdolell M. Visual acuity assessment: a comparison of two tests for measuring children's vision. Ophthalmic and Physiological Optics 2003;23:541-6.

17. Hyvärinen L, Näsänen R, Laurinen P. New visual acuity test for pre-school children. Acta Ophthalmol 1980;58:507-11.

18. ETDRS Manual of Operations. In: ETDRS Coordinating Center University of Maryland. Baltimore M, editor. Baltimore:: National Technical Information Service Publication; 1985.

19. Atkinson J, Braddick O. Assessment of visual acuity in infancy and early childhood. Acta Ophthalmologica 1983;61:18-26.

20. Atkinson J, Anker S, Evans C, Hall R, Pimm-Smith E. Visual acuity testing of young children with the Cambridge Crowding Cards at 3 and $6 \mathrm{~m}$. Acta Ophthalmologica 1988;66:505-8.

21. Woodruff ME. Observations on the visual acuity of children during the first five years of life. American journal of optometry and archives of American Academy of Optometry 1972;49:205.

22. Sheridan MD. What Is Normal Distance Vision at Five to Seven Years?

Developmental Medicine \& Child Neurology 1974;16:189-95. 
23. Smørvik DAG, Bosnes O. Assessment of visual acuity in preschool children. Scandinavian Journal of Psychology 1976;17:122-4.

24. Simons K. Visual acuity norms in young children. Survey of Ophthalmology $1983 ; 28: 84$

25. Semenov LA, Chernova ND, Bondarko VM. The measurement of visual acuity and the crowding effect in children from the age of 3 to 9. Fiziologiia Cheloveka 2000;26:21.

26. Jeon ST, Hamid J, Maurer D, Lewis TL. Developmental changes during childhood in single-letter acuity and its crowding by surrounding contours. Journal of Experimental Child Psychology 2010;107:423-37.

27. Pan Y, Tarczy-Hornoch K, Cotter Susan A, Wen G, Borchert MS, Azen SP, Varma R. Visual Acuity Norms in Preschool Children: The Multi-Ethnic Pediatric Eye Disease Study. Optometry and Vision Science: official publication of the American Academy of Optometry 2009;86:607.

28. Sonksen PM, Wade AM, Proffitt R, Heavens S, Salt AT. The Sonksen logMAR test of visual acuity: II. Age norms from 2 years 9 months to 8 years. Journal of AAPOS 2008;12:18-22.

29. Simmers AJ, Gray LS, Spowart K. Screening for amblyopia: a comparison of paediatric letter tests. In: British Journal of Ophthalmology; 1997. p. 465-9.

30. Morad Y, Werker E, Nemet P. Visual acuity tests using chart, line, and single optotype in healthy and amblyopic children. Journal of American Association for Pediatric Ophthalmology and Strabismus 1999;3:94-7.

31. Sheridan MD. Sheridan-Gardiner test for visual acuity. British Medical Journal $1970 ; 2: 108$.

32. Lippmann O. Vision of young children. Archives of Ophthalmology 1969;81:763. 
33. Rozhkova GI, Podugolnikova TA, Vasiljeva NN. Visual acuity in 5-7-year-old children: individual variability and dependence on observation distance. Ophthalmic and Physiological Optics 2005;25:66-80.

34. Bondarko VM, Semenov LA. Visual acuity and the crowding effect in 8-to 17year-old schoolchildren. Human Physiology 2005;31:532-8.

35. Shah N, Laidlaw DAH, Graham B, Chloe R. Effect of letter separation on computerised visual acuity measurements: comparison with the gold standard Early Treatment Diabetic Retinopathy Study (ETDRS) chart. Ophthalmic and Physiological Optics 2010;30:200-3.

36. Elliott MC, Firth AY. The logMAR Kay picture test and the logMAR acuity test: a comparative study. Eye 2007;23:85-8.

37. Kooi FL, Toet A, Tripathy SP, Levi DM. The effect of similarity and duration on spatial interaction in peripheral vision. Spatial Vision 1994;8:255-80.

38. Mayer DL, Gross RD. Modified Allen pictures to assess amblyopia in young children. Ophthalmology 1990;97:827-32.

39. Kowler E, Martins AJ. Eye movements of preschool children. Science 1982;215:997.

40. Aring E, Grönlund MA, Hellström A, Ygge J. Visual fixation development in children. Graefe's Archive for Clinical and Experimental Ophthalmology 2007;245:1659-65.

41. Kay H. New method of assessing visual acuity with pictures. British Medical Journal; 1983;67:131-3. 


\section{Figure Legends}

Figure 1. The five tests used in the study. The left column from top to bottom shows the logMAR Crowded Test, the Sonksen logMAR Test and the Crowded Kay Picture Test. The right column shows the Sheridan Gardiner Test and the Single Kay Picture Test.

Figure 2. Normalized logMAR is plotted for each of the visual acuity tests to show the crowding effect. The unflanked single optotype logMAR results (either Sheridan Gardiner or Kay Pictures) were subtracted from the logMAR result of the respective crowded tests. The top panel shows data for the younger age group and the bottom panel for the older age group.

Figure 3. LogMAR is plotted as a function of age in months. The straight lines represent linear regression fits to each data set (acuity test). The top panel shows results of the Sheridan Gardiner Test (closed symbols and solid line), the Sonksen Test (open squares and dashed line) and the logMAR Crowded Test (cross symbols and dotted line). The bottom panel shows the results of the Single Kay Picture Test (closed symbols and solid line) and the Crowded Kay Picture Test (open symbols and dotted line). 


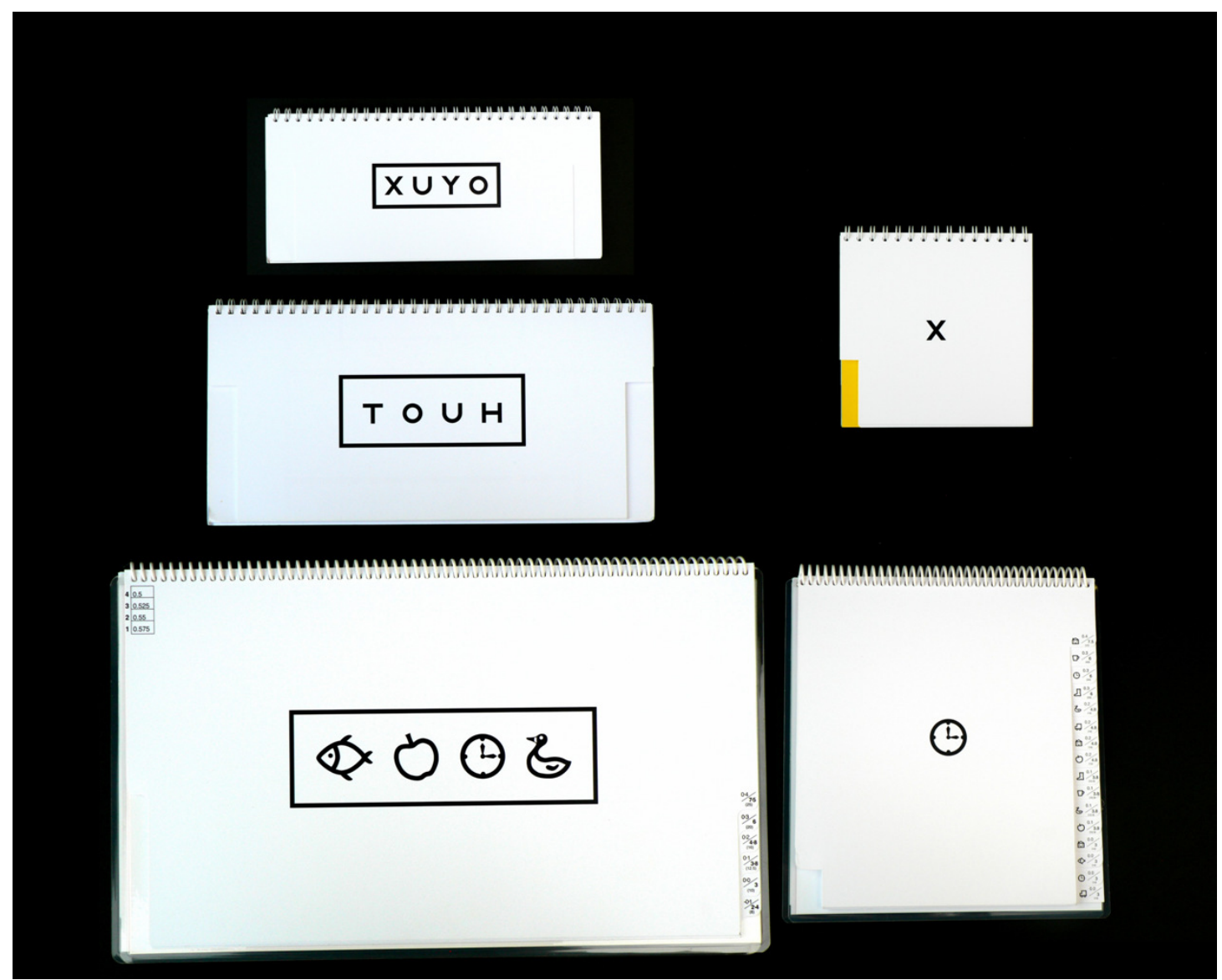



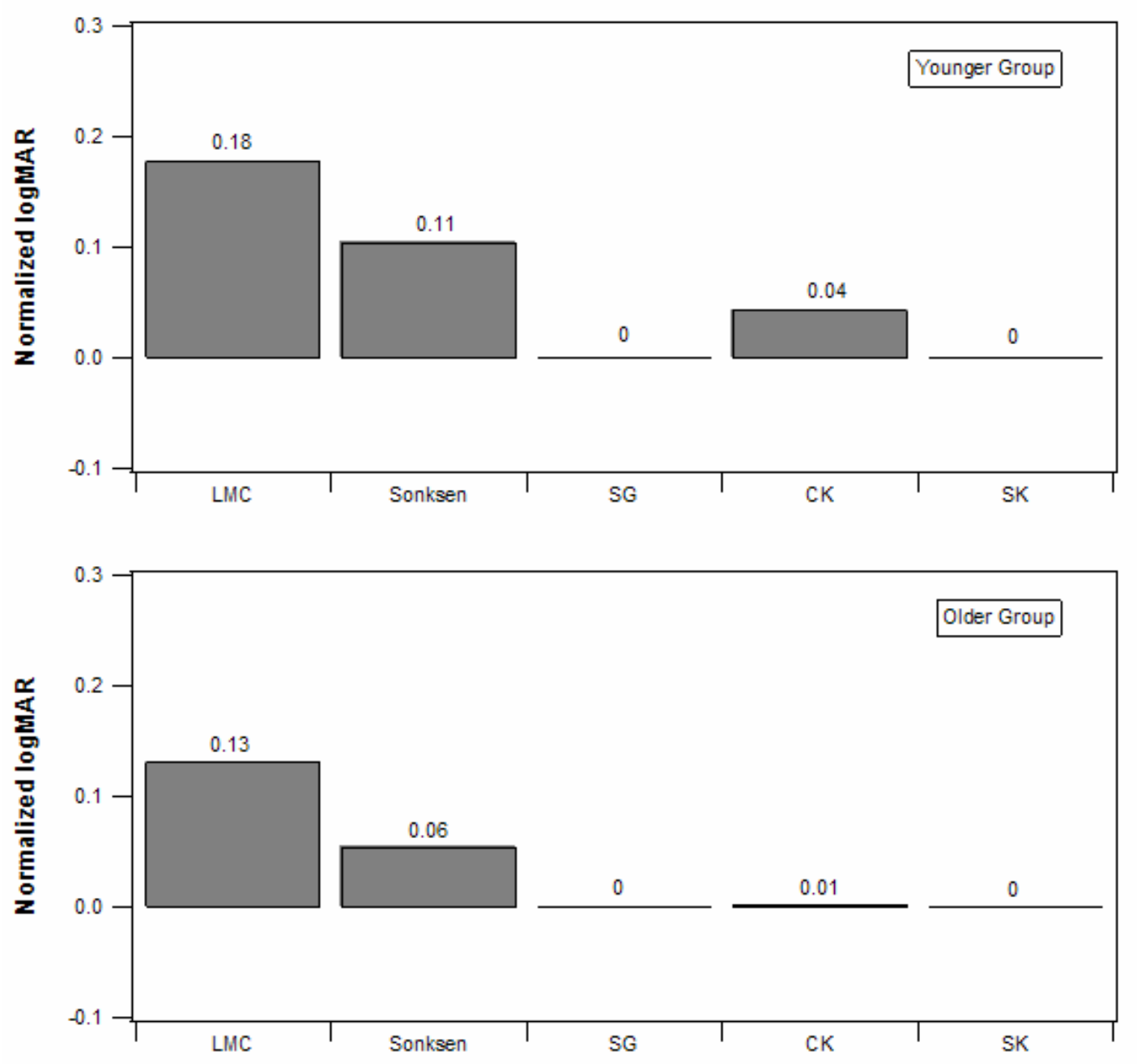

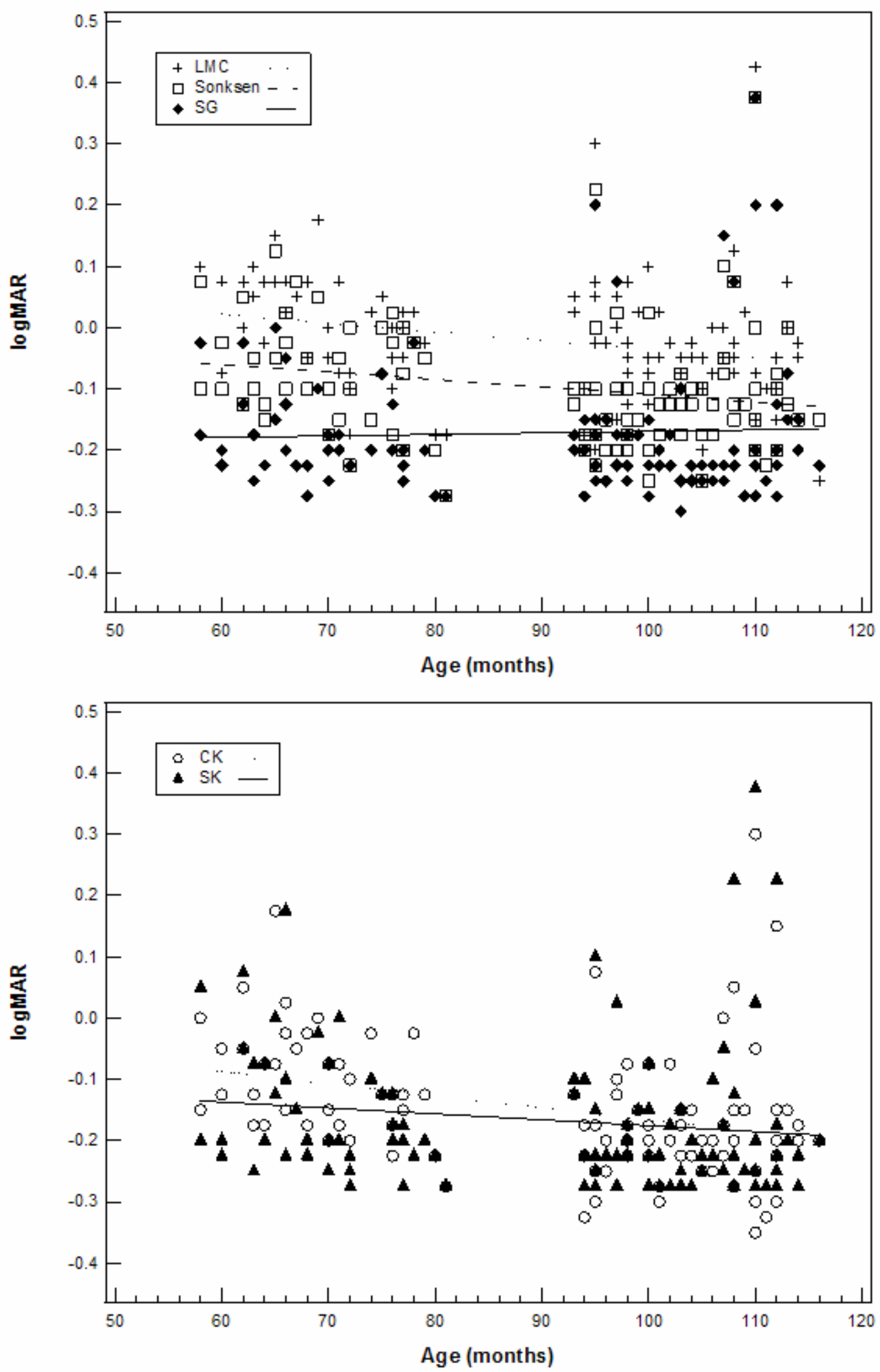
Table 1 Features of the 5 children's acuity tests used in the study.

\begin{tabular}{|l|c|c|c|c|c|}
\hline & $\begin{array}{c}\text { logMAR } \\
\text { Crowded } \\
\text { LMC }\end{array}$ & Sonksen & $\begin{array}{c}\text { Sheridan } \\
\text { Gardiner } \\
\text { SG }\end{array}$ & $\begin{array}{c}\text { Crowded } \\
\text { Kay } \\
\text { CK }\end{array}$ & $\begin{array}{c}\text { Single } \\
\text { Kay } \\
\text { SK }\end{array}$ \\
\hline $\begin{array}{l}\text { letters/ } \\
\text { symbols }\end{array}$ & X V O H U Y & X V O H U T & X V O H U T A & $\begin{array}{c}8 \text { Kay } \\
\text { pictures }\end{array}$ & $\begin{array}{c}8 \text { Kay } \\
\text { pictures }\end{array}$ \\
\hline optotypes/row & 4 & 4 & 1 & 4 & 1 \\
\hline $\begin{array}{l}\text { inter-optotype } \\
\text { spacing } \\
\text { optotype } \\
\text { units) }\end{array}$ & 0.5 & 1.0 & none & 0.5 & none \\
\hline $\begin{array}{l}\text { testing } \\
\text { distance used } \\
\text { in study }\end{array}$ & $3 m$ & $3 m$ & $6 m$ & $6 m$ & $6 m$ \\
\hline $\begin{array}{l}\text { range of } \\
\text { acuities } \\
\text { (logMAR) }\end{array}$ & 0.8 to -0.3 & 0.8 to -0.3 & 1.0 to -0.3 & 0.7 to -0.4 & 0.7 to -0.3 \\
\hline
\end{tabular}

Table 2 Mean visual acuity for each test (logMAR), with standard deviation in brackets

\begin{tabular}{|l|c|c|c|c|c|}
\hline & LMC & S & SG & CK & SK \\
\hline $\begin{array}{l}\text { Younger } \\
\text { children }\end{array}$ & $0.00(0.08)$ & $-0.07(0.09)$ & $-0.18(0.08)$ & $-0.10(0.09)$ & $-0.15(0.11)$ \\
\hline $\begin{array}{l}\text { Older } \\
\text { children }\end{array}$ & $-0.04(0.11)$ & $-0.11(0.11)$ & $-0.17(0.14)$ & $-0.17(0.11)$ & $-0.18(0.13)$ \\
\hline
\end{tabular}

LMC, logMAR Crowded; S, Sonksen; SG, Sheridan Gardiner; CK, Crowded Kay

Picture; SK, Single Kay Picture 